\title{
LETTER
}

\section{The burden of high-risk surgery and the potential benefit of goal-directed strategies}

\author{
Frederic Michard* \\ See related research by Cannesson et al., http://ccforum.com/content/15/4/R197
}

The survey by Cannesson and colleagues [1] in the previous issue of Critical Care shows that only around $16 \%$ of anesthetists $(5.4 \%$ of 210 US respondents and $30.4 \%$ of 158 European respondents) use a specific treatment protocol (that is, follow a goal-directed strategy) for the peri-operative hemodynamic management of patients undergoing high-risk surgery. In 2008, Weiser and colleagues [2] estimated the global volume of surgery to be 234.2 million procedures a year. According to Pearse and colleagues [3], high-risk surgical procedures represent around $12.5 \%$ of this total. A meta-analysis of the 29 randomized controlled trials investigating the value of peri-operative goal-directed strategies reported an average mortality rate of $9.4 \%$ in control groups and a significantly reduced mortality rate of $5.9 \%$ when a goaldirected strategy was adopted [4]. When putting all the pieces of this puzzle together, one can estimate that around 860,000 lives could potentially be saved every year (the equivalent of one life every 37 seconds) if such strategies became the standard of care around the world (Table 1).

In addition, millions of post-operative complications could be avoided. Indeed, the meta-analysis by Hamilton and colleagues [4] suggests that the post-operative complication rate could be reduced from $29.8 \%$ to $18.0 \%$ with goal-directed strategies. Given the potential volume of complications after high-risk surgery (Table 1), the direct costs of treating these complications as well as the indirect costs related to prolonged hospital length of stay are difficult to quantify precisely but without question are astronomically high. It may be time for heath-care systems and governments to consider peri-operative goal-directed strategies as part of quality improvement programs and as national priorities.

\section{Competing interests}

The author is a vice president of global medical strategy at Edwards Lifesciences (Irvine, CA, USA). The statements in this letter do not support the use of a specific treatment protocol or of a specific medical device for perioperative goal-directed strategies.

Published: 20 October 2011

\section{References}

1. Cannesson M, Pestel G, Ricks C, Hoeft A, Perel A: Hemodynamic monitoring and management in patients undergoing high-risk surgery: a survey among North American and European anesthesiologists. Crit Care 2011, 15:R197.

2. Weiser TG, Regenbogen SE, Thompson KD, Haynes AB, Lipsitz SR, Berry WR, Gawande AA: An estimation of the global volume of surgery: a modeling strategy based on available data. Lancet 2008, 372:139-144.

3. Pearse RM, Harrison DA, James P, Watson D, Hinds C, Rhodes A, Grounds RM, Bennett ED: Identification and characterization of the high-risk surgical population in the United Kingdom. Crit Care 2006, 10:R81.

4. Hamilton MA, Cecconi M, Rhodes A: A systematic review and meta-analysis on the use of preemptive hemodynamic intervention to improve postoperative outcomes in moderate and high-risk surgical patients. Anesth Analg 2011, 112:1392-1402.

doi:10.1186/cc10473

Cite this article as: Michard F: The burden of high-risk surgery and the potential benefit of goal-directed strategies. Critical Care 2011, 15:447.
*Correspondence: michard.frederic@free.fr

Critical Care, Edwards Lifesciences, Edwards Way, Irvine, CA 92614, USA 
Table 1. Estimates of the potential worldwide benefit of peri-operative goal-directed strategies

\begin{tabular}{lcc}
\hline & Yearly estimation & Reference \\
\hline All surgical procedures & $234,200,000$ & Weiser et al. [2] \\
High-risk surgical procedures (12.5\%) & $29,275,000$ & Pearse et al. [3] \\
$\quad$ High-risk procedures without GDS (84\%) & $24,591,000$ & Cannesson et al. [1] \\
$\quad$ Deaths without GDS (9.4\%) & $2,311,554$ & Hamilton et al. [4] \\
Deaths if GDS were to be adopted (5.9\%) & $1,450,869$ & Hamilton et al. [4] \\
$\quad$ Lives potentially saved if GDS were to be adopted & 860,685 & - \\
Complications without GDS (29.8\%) & $7,328,118$ & Hamilton et al. [4] \\
Complications if GDS were to be adopted (18.0\%) & $4,426,380$ & Hamilton et al. [4] \\
Complications potentially avoided if GDS were to be adopted & $2,901,738$ & - \\
\hline
\end{tabular}

GDS, goal-directed strategies. 